\title{
Inverse Scattering for Soft Fault Diagnosis in Electric Transmission Lines
}

\author{
Qinghua Zhang, Michel Sorine and Mehdi Admane
}

\begin{abstract}
Today's advanced reflectometry methods provide an efficient solution for the diagnosis of electric transmission line hard faults (open and short circuits), but they are much less efficient for soft faults, in particular, for faults resulting in spatially smooth variations of characteristic impedance. This paper attempts to fill an important gap for the application of the inverse scattering transform to reflectometry-based soft fault diagnosis: it clarifies the relationship between the reflection coefficient measured with reflectometry instruments and the mathematical object of the same name defined in the inverse scattering theory, by reconciling finite length transmission lines with the inverse scattering transform defined on the infinite interval. The feasibility of this approach is then demonstrated by numerical simulation of lossless transmission lines affected by soft faults, and by the solution of the inverse scattering problem effectively retrieving smoothly varying characteristic impedance profiles from reflection coefficients.
\end{abstract}

Index Terms - inverse scattering, fault diagnosis, transmission line, telegrapher's equations, Zakharov-Shabat equations.

\section{INTRODUCTION}

The fast development of electronic devices in modern engineering systems and in consumer products comes with an increasing number of electric wires in these equipments, and also inevitably with more and more failures related to electric connections. This fact has motivated research projects on methods for the diagnosis of faults in electric transmission lines. In this context, the technology of reflectometry has been extensively studied by different research groups [1], [2]. It consists in injecting electric signals from one end or from both ends of a line and in analyzing the reflected electric waves. At least for laboratory experimentations, currently this technology is able to detect and to locate hard faults (open circuit or short circuit) up to an accuracy of about $10 \mathrm{~cm}$. For soft faults, however, no satisfactory result has been reported, to our knowledge.

If the term "hard fault" refers to (almost) open or short circuits, any other kind of conduction faults may be qualified "soft". In [3] the difficulty for detecting soft faults has been investigated, mainly assuming weak (but abrupt) characteristic impedance changes caused by soft faults (such faults are called "frays" in [3]). The reported results show that wave reflections caused by the discontinuities at the borders of fray segments are comparable to, or even smaller than, the effects of noises and disturbances (cable movements, vibrations, etc.). For this reason, it seems impossible to detect and to locate such faults with reflectometry-based methods in real application environments.

This work has been supported by the ANR SEEDS project (Smart Embedded Electronic Diagnosis Systems).

Q.Z. and M.S. are with INRIA, Domaine de Voluceau-Rocquencourt, 78153 Le Chesnay Cedex, France. Q.Z. is also with IRISA, Campus de Beaulieu, 35042 Rennes, France. M.A. was with INRIA during his work on this project. Emails: zhang@irisa.fr, Michel.Sorine@inria.fr.
In this paper, the considered soft faults correspond to spatially smooth and arbitrary variations of characteristic impedance. Though there is no impedance discontinuity when such a fault occurs, the (smooth) impedance inhomogeneity can cause wave reflections. In addition to the difficulty related to the weakness of such reflections, it is conceptually difficult to deal with such faults, because they cannot be located in terms of impedance discontinuities. Moreover, no particular form of the characteristic impedance variations is assumed a priori. It is clear that any reflectometry-based method aiming at detecting and locating impedance discontinuities is not suitable for this problem.

The purpose of this paper is not to handle the problem reported in [3], namely weak reflections of soft faults drowned out by noises and disturbances. Instead, the particular case of "smooth soft faults" are investigated, under the assumption that noises and disturbances are limited by well controlled experimental conditions. Though this assumption is not realistic in many applications, the considered non-trivial problem merits investigations, while waiting for a satisfactory solution to noise and disturbance attenuation. Indeed, by making experiments at the ends of a transmission line, it is obviously difficult to inspect spatially smooth and arbitrary variations of characteristic impedance.

If computing reflection coefficients from specified transmission line characteristics is called a direct problem, its inverse problem corresponds to the purpose of this paper, namely retrieving distributed characteristics of a transmission line from reflection coefficients. This problem is related to the inverse scattering theory. Some early investigation on this topic was reported in [4]. It then attracted the attention of more researchers in 1970's and 1980's. For instance, time domain approaches were studied with continuous and discrete transmission line models respectively in [5] and [6]. The frequency domain inverse scattering approach is adopted in this paper for its direct connection with the measurements of frequency domain network analyzers. By transforming the telegrapher's equations to Schrödinger equation and to Zakharov-Shabat equations, Jaulent's pioneer work [7] has founded the theoretic basis for the inverse scattering problem of transmission lines in frequency domain. More recently, multiconductor lines have been studied in [8], but uniform transmission lines were assumed.

Despite these theoretic results, mostly dated a quarter of a century ago, to our knowledge, no successful experiment on their application to real transmission line fault diagnosis has been reported. The studies presented in this paper constitute an important step towards practical applications. Following Jaulent's frequency domain approach [7], the first contribution of this paper is to clarify the relationship between the reflection coefficient measured in practice and the mathematical object of 
the same name in the inverse scattering theory, by reconciling finite length transmission lines with the inverse scattering transform defined on the infinite interval. The feasibility of this approach is then illustrated by numerical simulations of lossless transmission lines with smooth variations of characteristic impedances, and by the solution of the inverse scattering problem, which effectively retrieves the characteristic variations of the simulated transmission lines.

Only lossless transmission lines are considered in this paper. In practice, the lossless assumption is reasonable for high quality transmission lines of moderate length. The more general lossy case, shortly discussed in Section IV, is currently under study. Some preliminary results will be reported in [9].

This paper is organized as follows. Section II is about the formulation of the inverse scattering problem for transmission lines, with, in particular, Section II-D clarifying the relationship between the reflection coefficient measured with reflectometry instruments and the mathematical object of the same name defined in the inverse scattering theory. Simulation results are presented in Section III. Concluding remarks are made in Section IV.

\section{TRANSMISSION LINE AND INVERSE SCATTERING}

This section is devoted to the transformations of the telegrapher's equations leading to the Zakharov-Shabat equations and the related inverse scattering problem. The basic result has been established by Jaulent [7], but it was not clear how to reconcile finite length transmission lines with the inverse scattering transform defined on the infinite interval. The main purpose of this section is to fill this gap.

\section{A. The telegrapher's equations and the reflection coefficient}

In this subsection the telegrapher's equations for lossless transmission lines will be recalled, so will the reflection coefficient as defined in electric engineering.

Consider a lossless transmission line connected to an alternating voltage source of frequency $k$ at the left end and to a load at the right end, as illustrated in Figure 1 ( $k$ will be treated as a wavenumber in the inverse scattering problem). Let $L(z)$ and $C(z)$ be respectively the distributed inductance and capacitance along the longitudinal axis $z$ of the transmission line, then, following [7], the voltage $V(k, z)$ and the current $I(k, z)$ at any point $z$ along the longitudinal axis of the line are governed by the telegrapher's equations

$$
\begin{aligned}
& \frac{d}{d z} V(k, z)-i k L(z) I(k, z)=0 \\
& \frac{d}{d z} I(k, z)-i k C(z) V(k, z)=0
\end{aligned}
$$

where $i$ is the imaginary unit.

In Figure 1, if the source connected to the left end of the transmission line is an instrument for reflection coefficient measurement, typically a network analyzer, then the measured reflection coefficient is

$$
r_{e}(k)=\frac{Z\left(k, z_{S}\right)-Z_{S}}{Z\left(k, z_{S}\right)+Z_{S}}
$$

where

$$
Z\left(k, z_{S}\right)=\frac{V\left(k, z_{S}\right)}{I\left(k, z_{S}\right)}
$$

is the input impedance of the transmission line, and $Z_{S}$ is the internal impedance of the source.

In textbooks on transmission lines, reflection coefficients are often defined at the load end or at the source end of uniform transmission lines, such as in [10, page 17] and in [11, page 193]. These reflection coefficients characterize more the adaptation of the load or the source than the transmission line itself. The reflection coefficient $r_{e}(k)$ expressed in (2) characterizes better the transmission line. Its definition is related to the S-parameters of transmission lines [12, chapter 13].

The quantity $r_{e}(k)$, as a function of $k$, will be referred to as engineering reflection coefficient in this paper, as it corresponds to a measurement frequently used in engineering practice.

\section{B. From telegrapher's equations to Zakharov-Shabat equa- tions}

To connect the telegrapher's equations to the scattering theory, the first step is to replace the space coordinate $z$ by the wave propagation time through the Liouville transformation

$$
x(z)=\int_{0}^{z} \sqrt{L(s) C(s)} d s .
$$

As $1 / \sqrt{L(s) C(s)}$ is the wave propagation velocity at the point $s, x(z)$ is clearly the wave propagation time from the position 0 to the position $z$.

After this coordinate change from $z$ to $x$, by abuse of notation, $L(z(x))$ will be simply written as $L(x)$, and similarly for $C(x), V(k, x), I(k, x)$. Then the telegrapher's equations (1) become

$$
\begin{aligned}
& \frac{d V(k, x)}{d x}=i k Z_{0}(x) I(k, x) \\
& \frac{d I(k, x)}{d x}=i k Z_{0}^{-1}(x) V(k, x)
\end{aligned}
$$

with

$$
Z_{0}(x)=\sqrt{\frac{L(x)}{C(x)}}
$$

being the characteristic impedance of the lossless transmission line at the point $x$. Accordingly, the circuit of Figure 1 is redrawn, by simply replacing $z, z_{S}$ and $z_{L}$ respectively by $x$, $x_{S}=x\left(z_{S}\right)$ and $x_{L}=x\left(z_{L}\right)$, as shown in Figure 2.

In the new coordinate system, define the reflected and incident waves ${ }^{1}$

$$
\begin{aligned}
& \nu_{1}(k, x)=\frac{1}{2}\left(Z_{0}^{-\frac{1}{2}}(x) V(k, x)-Z_{0}^{\frac{1}{2}}(x) I(k, x)\right) \\
& \nu_{2}(k, x)=\frac{1}{2}\left(Z_{0}^{-\frac{1}{2}}(x) V(k, x)+Z_{0}^{\frac{1}{2}}(x) I(k, x)\right)
\end{aligned}
$$

\footnotetext{
${ }^{1}$ The pair $\nu_{1}(k, x)-\nu_{2}(k, x)$ defined here is slightly different from the corresponding notation $Y$ in [7], to be better in agreement with the definition of scattering parameters in engineering practice. Accordingly, a negative sign is also added to the potential function $q(x)$ defined in (9).
} 
Some direct computations from (5) and (7) then lead to

$$
\begin{aligned}
& \frac{d \nu_{1}(k, x)}{d x}+i k \nu_{1}(k, x)=q(x) \nu_{2}(k, x) \\
& \frac{d \nu_{2}(k, x)}{d x}-i k \nu_{2}(k, x)=q(x) \nu_{1}(k, x)
\end{aligned}
$$

with

$$
q(x)=-\frac{1}{4} \frac{d}{d x}\left[\ln \frac{L(x)}{C(x)}\right]=-\frac{1}{2 Z_{0}(x)} \frac{d}{d x} Z_{0}(x) .
$$

These computations have implicitly used the following assumption.

Assumption 1: For $x$ ranging within the transmission line, the functions $L(x)$ and $C(x)$ are such that the characteristic impedance $Z_{0}(x)$ as defined in (6) is differentiable.

Equations (8), known as Zakharov-Shabat equations, constitute the main mathematical object connecting the electric transmission line to the scattering theory. The related inverse scattering transform computes the so-called potential function $q(x)$ from the reflection coefficient (in a sense to be recalled in this paper). It is clear that the characteristic impedance along the $x$-axis as defined in equation (6) can be determined from this potential function $q(x)$ through the ratio $L(x) / C(x)$. Hence the inverse scattering transform provides a powerful mathematical tool for the monitoring of characteristic impedance variations in electric transmission lines.

\section{Jost solutions of the Zakharov-Shabat equations and the theoretic reflection coefficient}

In this subsection recalling some concepts of the scattering theory, the pair $\nu_{1}(k, x)$ and $\nu_{2}(k, x)$ denotes a solution of the Zakharov-Shabat equations (8), and for the moment let us forget their relationship with $V(k, x)$ and $I(k, x)$ through (7).

In [13]-[15], inverse scattering problems are studied for systems modeled by equations (8) with $x$ ranging over the infinite interval $(-\infty,+\infty)$. It is assumed that the potential function $q(x)$ involved in (8) decays sufficiently fast when $x \rightarrow \pm \infty$, so that the solutions of the Zakharov-Shabat equations (8) have their limiting behaviors expressed in terms of $\exp ( \pm i k x)$.

Consider a particular solution of (8) (not necessarily given by (7)) with its limiting behaviors satisfying

$$
\begin{aligned}
& \lim _{x \rightarrow+\infty} \nu_{1}(k, x)=0 \\
& \lim _{x \rightarrow-\infty} \nu_{2}(k, x) \exp (-i k x)=1
\end{aligned}
$$

This solution is known as a Jost solution. Its left limiting behavior defines a reflection coefficient $r_{\text {th }}(k)$ :

$$
r_{\text {th }}(k)=\lim _{x \rightarrow-\infty} \frac{\nu_{1}(k, x)}{\nu_{2}(k, x)} \exp (2 i k x)
$$

This coefficient $r_{\text {th }}(k)$ is sometimes referred to as the left reflection coefficient, because it represents the ratio between the incident wave $\nu_{2}(k, x)$ coming from $-\infty$ and the reflected wave $\nu_{1}(k, x)$. Similarly, the right reflection coefficient can also be defined.

Through the inverse scattering transform, the potential function $q(x)$ can be retrieved from $r_{\text {th }}(k)$ [13]-[15]. This quantity

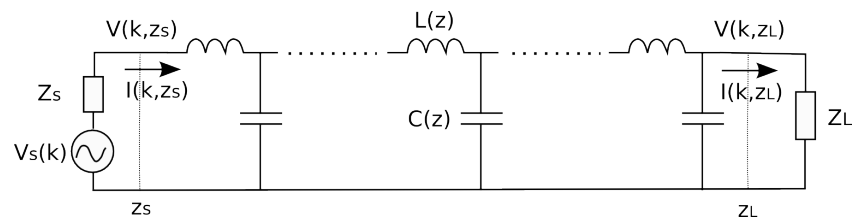

Fig. 1. A transmission line connected to a voltage source and a load, illustrated in $z$-coordinate. The source internal impedance $Z_{S}=Z_{0}\left(z_{S}\right)$ and the load impedance $Z_{L}=Z_{0}\left(z_{L}\right)$ are locally matched to the characteristic impedance of the line.

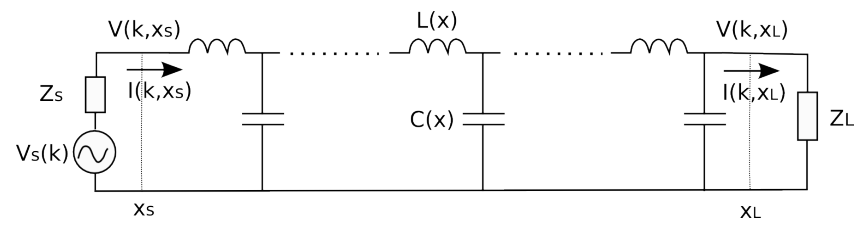

Fig. 2. The circuit of Figure 1 re-illustrated in $x$-coordinate.

$r_{\text {th }}(k)$, defined with the limiting behaviors of a Jost solution of the Zakharov-Shabat equations, will be referred to as the theoretic reflection coefficient in this paper.

\section{The relationship between the two reflection coefficient definitions}

The purpose of this subsection is to clarify the relationship between the definitions of the engineering reflection coefficient (2) and the theoretic reflection coefficient (11).

The theoretic reflection coefficient $r_{\text {th }}(k)$ has been defined with a solution of the Zakharov-Shabat equations for $x$ ranging from $-\infty$ to $+\infty$. To relate this definition to the finite length transmission line circuit illustrated in Figure 2, the circuit will be replaced by an equivalent circuit as specified in the following proposition.

Proposition 1: Build a new circuit, as shown in Figure 3, by modifying the circuit of Figure 2 as follows:

- insert a uniform transmission line of length $a$ with characteristic impedance $Z_{0}(x)=Z_{S}$ between the source and the left end;

- insert a uniform transmission line of length $b$ with characteristic impedance $Z_{0}(x)=Z_{L}$ between the right end and the load;

- add a phase shift $-k a$ to the source voltage.

Then, for any positive values of $a$ and $b$, the new circuit of Figure 3 and the one of Figure 2 are equivalent, in the sense that they have the same values of $V(k, x)$ and $I(k, x)$ for any $x \in\left[x_{S}, x_{L}\right]$.

Proof of Proposition 1. See the Appendix at the end of this paper.

As this proposition holds for any (arbitrarily large) positive values of $a$ and $b$, the quantities $V(k, x), I(k, x)$ and $Z_{0}(x)$ of the extended circuit are virtually defined for all $x \in \mathbb{R}$, so are the related $\nu_{1}(k, x), \nu_{2}(k, x)$ and $q(x)$.

To ensure well defined potential function $q(x)$ as formulated in (9) at the two connection points $x_{S}$ and $x_{L}$ in the extended 


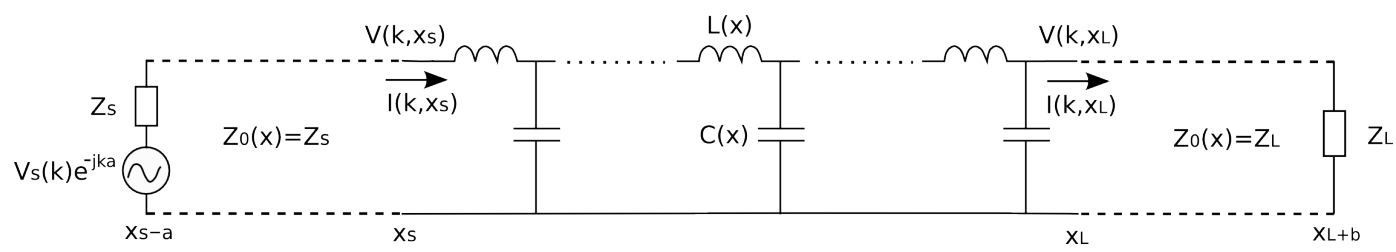

Fig. 3. The same transmission line as in Figure 2, but now the source is connected through a uniform line of length $a$ with characteristic impedance $Z_{0}(x)=Z_{S}$, the load is connected through a uniform line of length $b$ with characteristic impedance $Z_{0}(x)=Z_{L}$, and the source phase is shifted by $-k a$.

circuit of Figure 3, the characteristic impedance $Z_{0}(x)$ should be smooth at these two points, implying the following assumption.

Assumption 2: The transmission line in Figure 2 is locally adapted at its left and right ends such that

$$
\lim _{x \rightarrow x_{S}^{+}} \frac{d Z_{0}(x)}{d x}=\lim _{x \rightarrow x_{L}^{-}} \frac{d Z_{0}(x)}{d x}=0
$$

and

$$
\begin{aligned}
& Z_{S}=Z_{0}\left(x_{S}\right) \triangleq \lim _{x \rightarrow x_{S}^{+}} Z_{0}(x) \\
& Z_{L}=Z_{0}\left(x_{L}\right) \triangleq \lim _{x \rightarrow x_{L}^{-}} Z_{0}(x) .
\end{aligned}
$$

Proposition 2: When $a$ and $b$ both tend to $+\infty$, for the circuit of Figure 3, the pair $\nu_{1}(k, x)$ and $\nu_{2}(k, x)$ formed by (7) constitutes a solution of the Zakharov-Shabat equations (8) for all $x \in \mathbb{R}$. The theoretic reflection coefficient $r_{\text {th }}(k)$ defined in (11) with the limiting behaviors of $\nu_{1}(k, x)$ and $\nu_{2}(k, x)$ is related to the engineering reflection coefficient $r_{e}(k)$ defined in (2) through the equality

$$
r_{\text {th }}(k)=r_{e}(k) \exp \left(2 i k x_{S}\right)
$$

Proof of Proposition 2. This proof will consist of the following steps: (i) The pair $\nu_{1}(k, x)$ and $\nu_{2}(k, x)$ is a solution of the Zakharov-Shabat equations for all $x \in \mathbb{R}$. (ii) The pair $\nu_{1}(k, x)$ and $\nu_{2}(k, x)$ is a Jost solution, up to a coefficient common to $\nu_{1}(k, x)$ and $\nu_{2}(k, x)$. (iii) The limit in the definition (11) of the theoretic reflection coefficient is reached at $x=x_{S}$. (iv) $r_{\text {th }}(k)=r_{e}(k) \exp \left(2 i k x_{S}\right)$.

All the transformations and equations (4)-(9) are valid for the circuit of Figure 3 with arbitrary $a$ and $b$. It means that the Zakharov-Shabat equations (8) are derived from the telegrapher's equations with $\nu_{1}(k, x)$ and $\nu_{2}(k, x)$ defined in (7) for all $x \in\left[x_{S}-a, x_{L}+b\right]$. It is then clear that the pair $\nu_{1}(k, x)$ and $\nu_{2}(k, x)$ is a solution of the Zakharov-Shabat equations (8) for $x \in\left[x_{S}-a, x_{L}+b\right]$. When $a$ and $b$ tend to $+\infty$, the solution is valid for $x \in \mathbb{R}$.

It will be shown in the following that the pair $\nu_{1}(k, x)$ and $\nu_{2}(k, x)$ satisfies the limiting conditions (10), up to a factor common to $\nu_{1}(k, x)$ and $\nu_{2}(k, x)$.

Let us first examine the value of $\nu_{1}(k, x)$ for any $x \in$ $\left[x_{L}, x_{L}+b\right]$. As the load $Z_{L}$ is matched to the extended uniform line, the equalities

$$
V(k, x)=Z_{0}(x) I(k, x)=Z_{L} I(k, x)
$$

hold for any $x \in\left[x_{L}, x_{L}+b\right]$. Therefore, following (7a),

$$
\begin{aligned}
\nu_{1}(k, x) & =\frac{1}{2}\left(Z_{0}^{-\frac{1}{2}}(x) Z_{0}(x) I(k, x)-Z_{0}^{\frac{1}{2}}(x) I(k, x)\right) \\
& =0
\end{aligned}
$$

for all $x \in\left[x_{L},+\infty\right)$ when $b \rightarrow+\infty$. It is then clear that the first limiting condition (10a) is satisfied.

Now consider the case of $x \in\left[x_{S}-a, x_{S}\right]$. As the characteristic impedance $Z_{0}(x)=Z_{S}$ is constant over the uniform line extension, the potential function $q(x)=0$, according to (9). Hence the Zakharov-Shabat equations (8) become

$$
\begin{aligned}
& \frac{d \nu_{1}(k, x)}{d x}+i k \nu_{1}(k, x)=0 \\
& \frac{d \nu_{2}(k, x)}{d x}-i k \nu_{2}(k, x)=0
\end{aligned}
$$

for all $x \in\left(-\infty, x_{S}\right]$, when $a \rightarrow+\infty$. These are two decoupled first order differential equations, whose solutions are the reflected and the incident waves

$$
\begin{aligned}
& \nu_{1}(k, x)=c_{1}(k) \exp (-i k x) \\
& \nu_{2}(k, x)=c_{2}(k) \exp (i k x)
\end{aligned}
$$

with some coefficients $c_{1}(k)$ and $c_{2}(k)$ independent of $x$.

As equation (16) holds for all $x \in\left(-\infty, x_{S}\right]$, the second limiting condition (10b) is satisfied up to the coefficient $c_{2}(k)$. The limiting condition (10) would be fully satisfied if $\nu_{1}(k, x)$ and $\nu_{2}(k, x)$ were both divided by $c_{2}(k)$. This normalization is not necessary in view of the definition of the theoretic reflection coefficient as formulated in (11), as $\nu_{1}(k, x)$ and $\nu_{2}(k, x)$ share the same factor. It is thus confirmed that the limiting behaviors of $\nu_{1}(k, x)$ and $\nu_{2}(k, x)$ are in agreement with the definition of the theoretic reflection coefficient.

Because (15) and (16) both hold for all $x \leq x_{S}$, the limiting value in the definition (11) of the theoretic reflection coefficient is reached at $x=x_{S}$. Hence this definition can be replaced by

$$
r_{\text {th }}(k)=\frac{\nu_{1}\left(k, x_{S}\right)}{\nu_{2}\left(k, x_{S}\right)} \exp \left(2 i k x_{S}\right)
$$

It then follows from (7) that

$$
\begin{aligned}
r_{\text {th }}(k) & =\frac{Z_{0}^{-\frac{1}{2}}\left(x_{S}\right) V\left(k, x_{S}\right)-Z_{0}^{\frac{1}{2}}\left(x_{S}\right) I\left(k, x_{S}\right)}{Z_{0}^{-\frac{1}{2}}\left(x_{S}\right) V\left(k, x_{S}\right)+Z_{0}^{\frac{1}{2}}\left(x_{S}\right) I\left(k, x_{S}\right)} \exp \left(2 i k x_{S}\right) \\
& =\frac{V\left(k, x_{S}\right)-Z_{0}\left(x_{S}\right) I\left(k, x_{S}\right)}{V\left(k, x_{S}\right)+Z_{0}\left(x_{S}\right) I\left(k, x_{S}\right)} \exp \left(2 i k x_{S}\right) \\
& =\frac{V\left(k, x_{S}\right)-Z_{S} I\left(k, x_{S}\right)}{V\left(k, x_{S}\right)+Z_{S} I\left(k, x_{S}\right)} \exp \left(2 i k x_{S}\right)
\end{aligned}
$$


where the last equality is due to the fact that $Z_{0}\left(x_{S}\right)=Z_{S}$ following (12).

Rewrite (3) as $Z\left(k, x_{S}\right)=V\left(k, x_{S}\right) / I\left(k, x_{S}\right)$, then

$$
r_{\text {th }}(k)=\frac{Z\left(k, x_{S}\right)-Z_{S}}{Z\left(k, x_{S}\right)+Z_{S}} \exp \left(2 i k x_{S}\right) .
$$

Compare this result with (2) by reminding that $Z\left(k, x_{S}\right)$ and $Z\left(k, z_{S}\right)$ denote the same quantity under different coordinate systems, the relationship between the two reflection coefficients expressed in (14) is then proved.

This result indicates that the engineering reflection coefficient $r_{e}(k)$ and the theoretic reflection coefficient $r_{\mathrm{th}}(k)$ are identical up to a phase shift of $2 k x_{S}$. It appears that the definition of $r_{\mathrm{th}}(k)$ depends on the choice of the origin of the $x$-axis. By choosing the origin such that $x_{S}=0$, the two reflection coefficients coincide exactly.

\section{E. Inverse scattering for characteristic impedance monitoring}

To retrieve the potential function $q(x)$ (and thus the characteristic impedance $Z_{0}(x)$ ) from the reflection coefficient only, it is assumed that the Zakharov-Shabat equations (8) have no bound state (square integrable solution for $x \in \mathbb{R}$ ), following [7].

The inverse scattering transform consists of the following steps for computing the potential function $q(x)$ and $L(x) / C(x)=Z_{0}^{2}(x)$, from the reflection coefficient $r_{\text {th }}(k)$ as defined in (11). See [13], [14] for more details.

1) Let the Fourier transform of the reflection coefficient $r_{\text {th }}(k)$ be

$$
\rho(x)=\frac{1}{2 \pi} \int_{-\infty}^{+\infty} r_{\text {th }}(k) \exp (-i k x) d k
$$

2) Solve the integral equations (known as Gel'fandLevitan-Marchenko equations) for its unknown kernels $A_{1}(x, y)$ and $A_{2}(x, y)$ :

$$
\begin{aligned}
& A_{1}(x, y)+\int_{-y}^{x} A_{2}(x, s) \rho(y+s) d s=0 \\
& A_{2}(x, y)+\rho(x+y)+\int_{-y}^{x} A_{1}(x, s) \rho(y+s) d s=0
\end{aligned}
$$

3) Compute the potential function $q(x)$ through

$$
q(x)=2 A_{2}(x, x)
$$

4) By inverting equation (9), compute

$$
\frac{L(x)}{C(x)}=\frac{L\left(x_{S}\right)}{C\left(x_{S}\right)} \exp \left(-4 \int_{x_{S}}^{x} q(s) d s\right)
$$

In practice, the integral equations have to be solved numerically, by discretizing the kernels $A_{1}(x, y)$ and $A_{2}(x, y)$ over a grid in the $x-y$ plane. By choosing the origin of the $x$-axis such that $x_{S}>0$, the potential $q(x)=0$ for $x<0$. It is then sufficient to compute the kernels $A_{1}(x, y)$ and $A_{2}(x, y)$ in the region $x \geq|y|$. The numerical inverse scattering algorithm used for producing the simulation results presented in the next section is the one of [16], which is an improved variant of the algorithm of [17]. Both algorithms produce similar results in our simulations, but the one of [16] is much faster.

\section{Simulation STUdY}

In this section, results of simulation will be presented to confirm the validity of the approach presented in this paper. The first step is to implement a numerical simulator generating the reflection coefficient from the specified profiles $L(z)$ and $C(z)$ of a transmission line.

\section{A. Transmission line simulator}

For the circuit of Figure 2, let

$$
Z(k, x)=\frac{V(k, x)}{I(k, x)}
$$

be the apparent impedance at any point $x \in\left[x_{S}, x_{L}\right]$. It is then straightforward to check that the telegrapher's equations (5) imply that $Z(k, x)$ satisfies the Riccati equation

$$
\frac{d}{d x} Z(k, x)=i k Z_{0}(x)-i k Z_{0}^{-1}(x) Z^{2}(k, x)
$$

where $Z_{0}(x)$ is the characteristic impedance as defined in (6).

For a given value of $k$, by initializing $Z(k, x)$ with the load impedance at the right end, namely

$$
Z\left(k, x_{L}\right)=Z_{L}
$$

equation (20) can be solved for $x<x_{L}$. In particular, the value $Z\left(k, x_{S}\right)$ at the left end of the transmission line is obtained. The reflection coefficient $r_{e}(k)$ is then computed with (2), and $r_{\text {th }}(k)$ with (14).

It then appears that the main computation of the simulator consists of the numerical solution of the Riccati equation (20) for the value of the apparent impedance $Z\left(k, x_{S}\right)$. This computation is repeated with different values of $k$ to cover a sufficiently large spectrum.

\section{B. Simulation results}

For the first example, a smoothly increasing $L(z)$ profile is simulated. The capacitance is kept to a constant value $C=0.1 \mathrm{nF} / \mathrm{m}$, and the corresponding ratio $L / C$ is depicted in Figure 4, in both $z$ and $x$ coordinates, where the units for $L / C$ are $\mu \mathrm{H} / \mathrm{nF}$. The simulated reflection coefficient $r_{\mathrm{th}}(k)$ (modulus and phase) is shown in Figure 5. The $L / C$ profile computed through the inverse scattering transform is compared to the original simulated profile in Figure 6.

Remark that the inverse scattering transform computes the ratio $L / C$ as a function of $x$. In practice it would be more useful to inspect the ratio as a function of $z$, the true spatial coordinate of the transmission line. Like all reflectometry methods, the information obtained by observing incident and reflected waves is related to the wave propagation time $x$. Without knowing the wave propagation velocity (varying along the line in the considered case), it is impossible to convert the result from $x$ to $z$. However, for moderate variations of the ratio $L / C$ (under the soft fault assumption), the two profiles in $x$ and in $z$ are similar, as shown in Figure 4 for the considered example. It is thus practically reasonable to assume a constant wave propagation velocity to convert the computed $L / C$ ratio to the $z$-coordinate. 

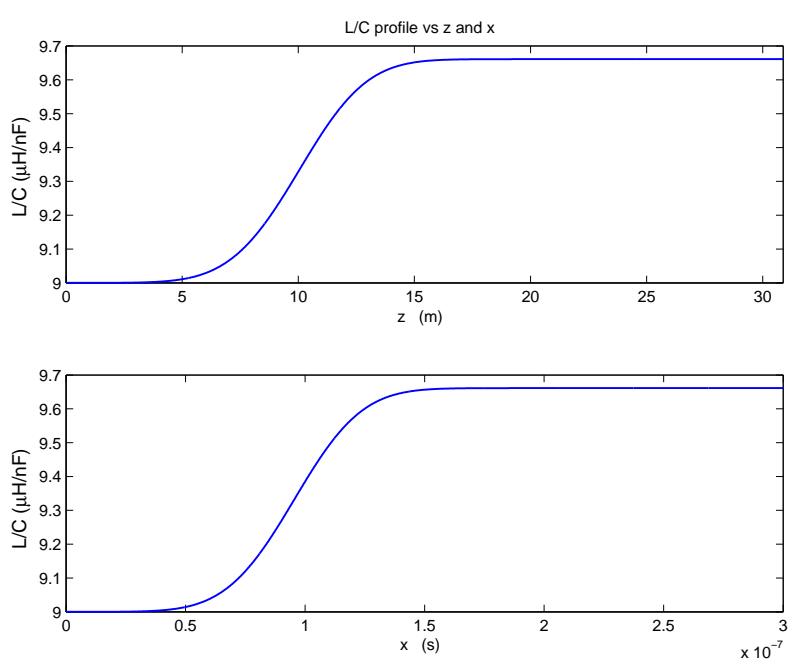

Fig. 4. Simulated smoothly increasing $L / C$ profile in $z$-and $x$-coordinates.
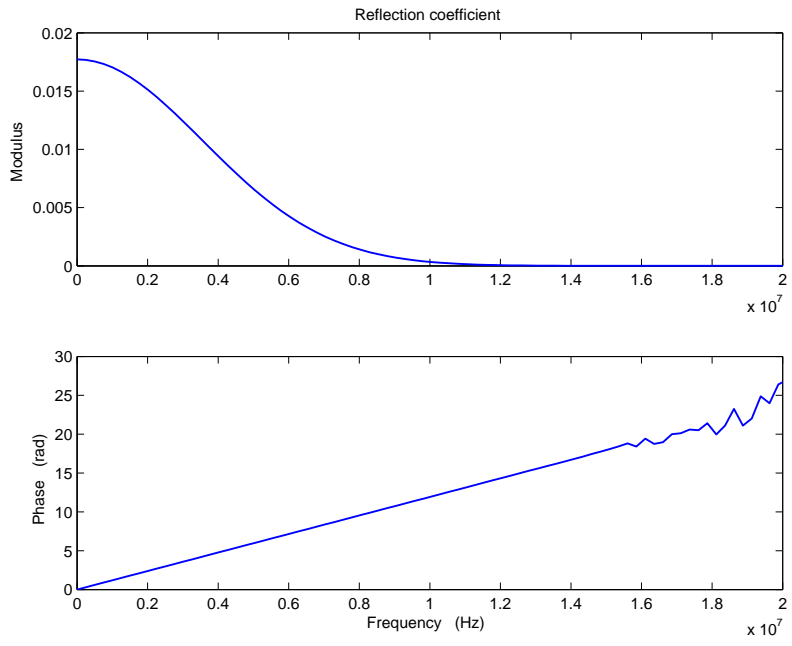

Fig. 5. Simulated reflection coefficient $r_{\text {th }}(k)$ for the smoothly increasing $L / C$ profile.

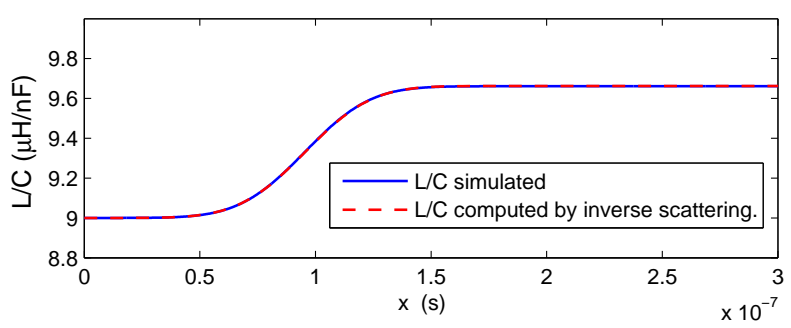

Fig. 6. The smoothly increasing $L / C$ profile computed by inverse scattering and compared to the simulated profile. The two curves may not be distinguishable if printed in black and white.
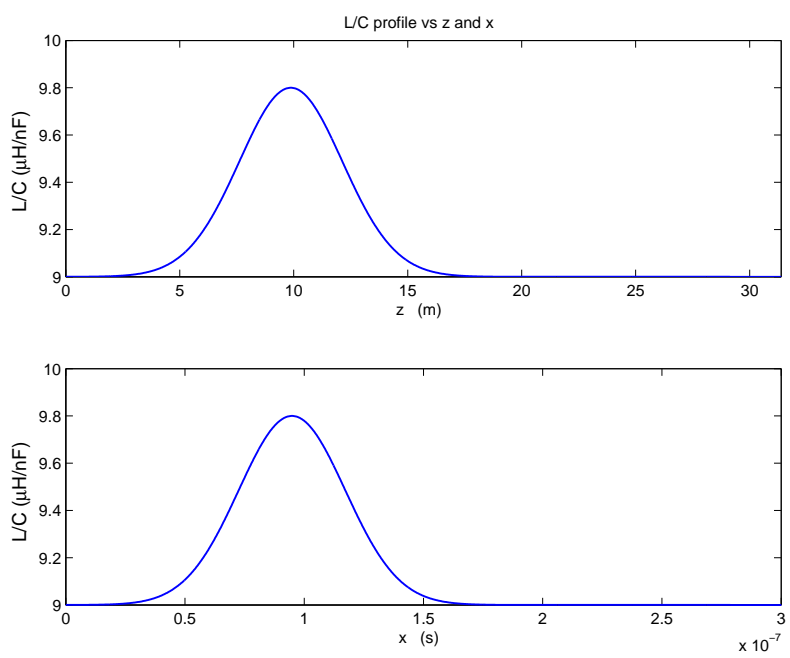

Fig. 7. Simulated hump-shaped $L / C$ profile in $z$ - and $x$-coordinates.
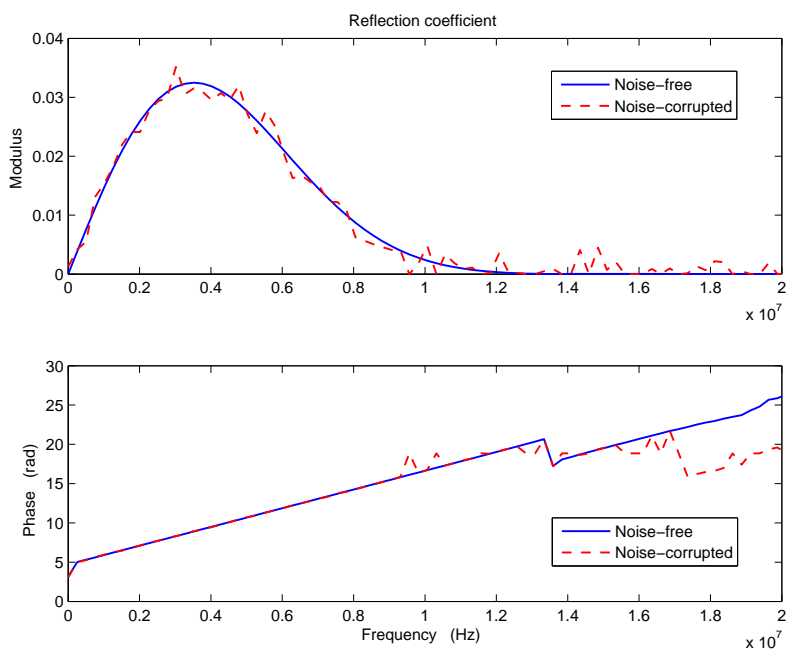

Fig. 8. Simulated reflection coefficients $r_{\text {th }}(k)$ for the hump-shaped $L / C$ profile, the noise-free case in solid line, and the noise-corrupted case in dashed line.

For the second example, a hump-shaped $L / C$ profile is simulated, as illustrated in Figure 7. The simulated reflection coefficient is plotted in Figure 8 (in solid line), and the $L / C$ profile computed by inverse scattering in Figure 9. Again the simulated $L / C$ profile is correctly retrieved by the inverse scattering transform.

The above simulation examples have been made under the

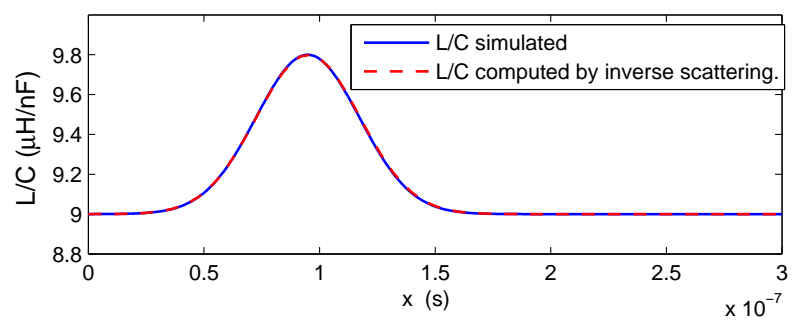

Fig. 9. The hump-shaped $L / C$ profile computed by inverse scattering and compared to the simulated profile. The two curves may not be distinguishable if printed in black and white. 


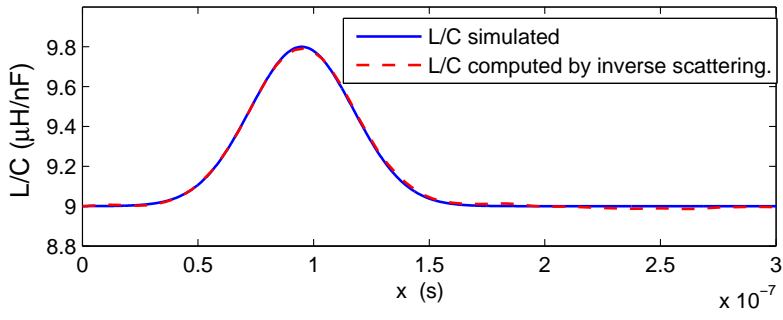

Fig. 10. The hump-shaped $L / C$ profile computed by inverse scattering from noise-corrupted reflection coefficient.

assumption of perfect model and noise-free measurement. To evaluate the robustness of the method to random uncertainties, noises can be added in the simulation study. The second simulation example is then repeated by adding a Gaussian noise to each simulated value of the reflection coefficient. The standard deviation of the added noise is equal to about $6 \%$ of the maximum absolute value of the simulated $r_{\mathrm{th}}(k)$. The noise-corrupted reflection coefficient (modulus and phase) is also illustrated in Figure 8 (dashed lines). The hump-shaped $L / C$ profile is again computed by inverse scattering, but now from the noise-corrupted reflection coefficient. The result is illustrated in Figure 10. Though the reflection coefficient has been significantly corrupted, the inverse scattering transform is still capable of retrieving reasonably the $L / C$ profile. This example is mainly to investigate the numerical stability of the inverse scattering method, not to simulate the disturbances in real application environments, like those studied in [3].

Though in theory the computation of the Fourier transform of the reflection coefficient requires the values of $r_{\text {th }}(k)$ for $k$ ranging from 0 to $+\infty$ (in (18) it ranges from $-\infty$ to $+\infty$, but $r_{\mathrm{th}}(-k)=r_{\mathrm{th}}^{*}(k)$, since $\rho(x)$ is real), in the above simulation examples the values of $r_{\text {th }}(k)$ are truncated to $20 \mathrm{MHz}$. The frequency bandwidth after truncation is related to the details of the characteristic impedance to be reconstructed: measurements of larger bandwidth capture more details.

\section{CONCLUSION}

Though the theoretic basis for the inverse scattering problem of electric transmission lines has been founded about a quarter of a century ago, there has been a gap between such theoretic results and their practical applications. By reconciling finite length transmission lines with the inverse scattering transform defined on the infinite interval, this paper constitutes an attempt to fill this gap. Moreover, the simulation studies reported in this paper confirm the feasibility of this approach.

The success of practical applications of this method will depend on the accuracy of the reflection coefficients used for solving the inverse problem. Though simulations have shown that the inverse scattering algorithm behaves reasonably when the reflection coefficients are slightly disturbed, in practice, various noises and disturbances may hide the effects of small faults. Some efficient noise and disturbance attenuation methods remain to be developed, so that methods for soft fault diagnosis, like the one presented in this paper, can be widely applied.
The lossless assumption made in this paper is only reasonable for high quality transmission lines of moderate length. For lossy transmission lines, as shown by Jaulent in [7], after the transformations adopted in this paper, an extra step transforms the telegrapher's equations for lossy transmission lines to a pair of Zakharov-Shabat equations similar to equations (8), but with two distinct potential functions in the two equations. Similar Gel'fand-Levitan-Marchenko integral equations can be established in this case [14]. Though the numerical algorithms solving these equations, like the one of [18], remain similar to those of the single-potential Zakharov-Shabat equations, their numerical stability in their applications to lossy transmission lines is more delicate. Some preliminary results of our studies on lossy transmission lines, extending the results presented in this paper, will be reported in [9].

\section{APPENDIX: PROOF OF PROPOSITION 1.}

In Figure 3, because the extended uniform line at the right side of $x_{L}$ is connected to the matched load $Z_{L}$, the total impedance of the extended line plus the matched load is equal to $Z_{L}$. Therefore, viewed from the left side of $x_{L}$, the extended line and the matched load behave as the simple load $Z_{L}$ of Figure 2.

When the telegrapher's equations (5) is applied to the circuit of Figure 3 for $x \in\left[x_{S}-a, x_{S}\right]$ where $Z_{0}(x)=Z_{S}$, the solution writes

$$
\begin{aligned}
V(k, x) & =\alpha(k) \exp (-i k x)+\beta(k) \exp (i k x) \\
I(k, x) & =\frac{1}{Z_{S}}[-\alpha(k) \exp (-i k x)+\beta(k) \exp (i k x)]
\end{aligned}
$$

in which $\alpha(k)$ and $\beta(k)$ are determined by the boundary conditions

$$
\begin{aligned}
& V_{S}(k) \exp (-i k a)-V\left(k, x_{S}-a\right)=Z_{S} I\left(k, x_{S}-a\right) \\
& V\left(k, x_{S}\right)=Z\left(k, z_{S}\right) I\left(k, x_{S}\right)
\end{aligned}
$$

with $Z\left(k, z_{S}\right)$ being the apparent impedance at the point $x_{S}$ of Figure 3, which is also equal to the input impedance of the circuit of Figure 2. It then yields

$$
\begin{aligned}
\alpha(k) & =\frac{1}{2} \frac{Z\left(k, z_{S}\right)-Z_{S}}{Z\left(k, z_{S}\right)+Z_{S}} V_{S}(k) \exp \left(i k x_{S}\right) \\
\beta(k) & =\frac{1}{2} V_{S}(k) \exp \left(-i k x_{S}\right)
\end{aligned}
$$

and

$$
\begin{aligned}
V\left(k, x_{S}\right) & =\frac{Z\left(k, z_{S}\right)}{Z\left(k, z_{S}\right)+Z_{S}} V_{S}(k) \\
I\left(k, x_{S}\right) & =\frac{1}{Z\left(k, z_{S}\right)+Z_{S}} V_{S}(k)
\end{aligned}
$$

On the other hand, for the circuit of Figure 2, the transmission line and the load $Z_{L}$ can be viewed from the source as an equivalent load of impedance $Z\left(k, z_{S}\right)$. With such an equivalent circuit, it is easy to compute the values of $V\left(k, x_{S}\right)$ and $I\left(k, x_{S}\right)$, which happen to coincide with those of equations (21) and (22). It means that $V\left(k, x_{S}\right)$ and $I\left(k, x_{S}\right)$ have the same values in Figure 2 and Figure 3. Consequently, for any $x \in\left[x_{S}, x_{L}\right], V(k, x)$ and $I(k, x)$ have the same values in these two circuits. 


\section{REFERENCES}

[1] P. Smith, C. Furse, and J. Gunther, "Analysis of spread spectrum time domain reflectometry for wire fault location," IEEE Sensors Journal, vol. 5, no. 6, pp. 1469-1478, December 2005.

[2] F. Auzanneau, M. Olivas, and N. Ravot, "A simple and accurate model for wire diagnosis using reflectometry," in PIERS Proceedings, August 2007.

[3] L. A. Griffiths, R. Parakh, C. Furse, and B. Baker, "The invisible fray: A critical analysis of the use of reflectometry for fray location," IEEE Sensors Journal, vol. 6, no. 3, pp. 697-706, June 2006.

[4] H. E. Moses and C. M. de Ridder, "Properties of dielectrics from reflection coefficients in one dimension," MIT Lincoln Laboratory, Tech. Rep. 322, 1963.

[5] B. Gopinath and M. M. Sondhi, "Inversion of the telegraph equation and the synthesis of nonuniform lines," Proceedings of the IEEE, vol. 59, no. 3, pp. 383- 392, 1971.

[6] A. M. Bruckstein and T. Kailath, "Inverse scattering for discrete transmission-line models," SIAM Review, vol. 29, no. 3, pp. 359-389, 1987.

[7] M. Jaulent, "The inverse scattering problem for LCRG transmission lines," Journal of Mathematical Physics, vol. 23, no. 12, pp. 2286-2290, December 1982.

[8] L. F. Knockaert, D. De Zutter, F. Olyslager, E. Laermans, and J. De Geest, "Recovering lossy multiconductor transmission line parameters from impedance or scattering representations," IEEE transactions on Advanced Packaging, vol. 25, no. 2, pp. 200-205, 2002.

[9] H. Tang and Q. Zhang, "Inverse scattering for lossy electric transmission line soft fault diagnosis," in IEEE International Symposium on Antennas and Propagation, Toronto, Canada, July 2010.

[10] L. N. Dworsky, Modern transmission line theory and applications. New York: John Wiley \& Sons, 1979.

[11] A. Weisshaar, "Transmission lines," in Handbook of Engineering Electromagnetics, R. Bansal, Ed. CRC Press, 2004, pp. 185-26.

[12] S. J. Orfanidis, Electromagnetic Waves and Antennas. http://www.ece.rutgers.edu/ orfanidi/ewa/, 2008.

[13] G. L. Lamb, Elements of Soliton Theory. New York: John Wiley \& Sons, 1980.

[14] W. Eckhaus and A. van Harten, The inverse scattering transformation and the theory of solitons: an introduction. Elsevier, 1981.

[15] R. J. Sobey and E. J. Colman, "Scattering analysis and synthesis of wave trains," Journal of the Australian Mathematical Society, vol. 25, pp. 44-63, 1983.

[16] G. Xiao and K. Yashiro, "An efficient algorithm for solving ZakharovShabat inverse scattering problem," IEEE Transactions on Antennas and Propagation, vol. 50, no. 6, pp. 807 -811, June 2002.

[17] P. V. Frangos and D. L. Jaggard, "A numerical solution to the ZakharovShabat inverse scattering problem," IEEE transactions on antennas and propagation, vol. 39, no. 1, pp. 74-79, 1991.

[18] , "Analytical and numerical solution to the two-potential ZakharovShabat inverse scattering problem," IEEE transactions on antennas and propagation, 1992. 